\title{
Ultrastable $\mathrm{CO}_{2}$ Laser Trapping of Lithium Fermions
}

\author{
K. M. O'Hara, S. R. Granade, M. E. Gehm, T. A. Savard, S. Bali, \\ C. Freed ${ }^{\dagger}$, and J. E. Thomas \\ Physics Department, Duke University, Durham, North Carolina 27708-0305
}

(March 8, 1999)

\begin{abstract}
We demonstrate an ultrastable $\mathrm{CO}_{2}$ laser trap that provides tight confinement of neutral atoms with negligible optical scattering and minimal laser noise induced heating. Using this method, fermionic ${ }^{6} \mathrm{Li}$ atoms are stored in a $0.4 \mathrm{mK}$ deep well with a 1/e trap lifetime of 300 seconds, consistent with a background pressure of $10^{-11}$ Torr. To our knowledge, this is the longest storage time ever achieved with an all-optical trap, comparable to the best reported magnetic traps.
\end{abstract}

PACS numbers: $32.80 . P j$

Copyright 1999 by the American Physical Society

Off-resonance optical traps have been explored for many years as an attractive means of tightly confining neutral atoms 1]. Far off resonance optical traps (FORTs) employ large detunings from resonance to achieve low optical heating rates and high density, as well as to enable trapping of multiple atomic spin states in nearly identical potentials [2 [6]. For $\mathrm{CO}_{2}$ laser traps [7], the extremely large detuning from resonance and the very low optical frequency lead to optical scattering rates that are measured in photons per atom per hour. Hence, optical heating is negligible. Such traps are potentially important for development of new standards and sensors based on spectroscopic methods, for precision measurements such as determination of electric dipole moments in atoms [8], and for fundamental studies of cold, weakly interacting atomic or molecular vapors.

However, all-optical atom traps have suffered from unexplained heating mechanisms that limit the minimum attainable temperatures and the maximum storage times in an ultrahigh vacuum [4, 9, 10]. Recently, we have shown that to achieve long storage times in all-optical traps that are not limited by optical heating rates, heating arising from laser intensity noise and beam pointing noise must be stringently controlled [11,12]. Properly designed $\mathrm{CO}_{2}$ lasers are powerful and extremely stable in both frequency and intensity 13,14, resulting in laser-noise-induced heating times that are measured in hours. Hence, in an ultrahigh vacuum (UHV) environment, where loss and heating arising from background gas collisions are minimized [15,16], extremely long storage times should be obtainable using ultrastable $\mathrm{CO}_{2}$ laser traps.

In this Letter, we report storage of ${ }^{6} \mathrm{Li}$ fermions in an ultrastable $\mathrm{CO}_{2}$ laser trap. Trap 1/e lifetimes of $300 \mathrm{sec}-$ onds are obtained, consistent with a background pressure of $10^{-11}$ Torr. This constitutes the first experimental proof of principle that extremely long storage times can be achieved in all-optical traps. Since arbitrary hyperfine states can be trapped, this system will enable exploration of s-wave scattering in a weakly interacting fermi gas.

The well-depth for a focused $\mathrm{CO}_{2}$ laser trap is determined by the induced dipole potential $U=-\alpha_{g} \overline{\mathcal{E}^{2}} / 2$, where $\alpha_{g}$ is, to a good approximation, the ground state static polarizability [7], and $\overline{\mathcal{E}}^{2}$ is the time average of the square of the laser field. In terms of the maximum laser intensity $I$ for the gaussian $\mathrm{CO}_{2}$ laser beam, the ground state well-depth $U_{0}$ in $\mathrm{Hz}$ is

$$
\frac{U_{0}}{h}(\mathrm{~Hz})=-\frac{2 \pi}{h c} \alpha_{g} I .
$$

In our experiments, a laser power of $\mathrm{P}=40 \mathrm{~W}$ typically is obtained in the trap region. A lens is used to focus the trap beam to field a $1 / \mathrm{e}$ radius of $a_{f}=50 \mu \mathrm{m}$, yielding an intensity of $I=2 P /\left(\pi a_{f}^{2}\right) \simeq 1.0 \mathrm{MW} / \mathrm{cm}^{2}$. For the I-P(20) line with $\lambda_{\mathrm{CO}_{2}} \simeq 10.6 \mu \mathrm{m}$, the Rayleigh length is $z_{0}=\pi a_{f}^{2} / \lambda_{\mathrm{CO}_{2}}=0.74 \mathrm{~mm}$. Using the Li ground state polarizability of $\alpha_{g}=24.3 \times 10^{-24} \mathrm{~cm}^{3}$ [17 yields a well depth of $U_{0} / h=-8 \mathrm{MHz}$, which is approximately $0.4 \mathrm{mK}$. For this tight trap, the ${ }^{6} \mathrm{Li}$ radial oscillation frequency is $4.7 \mathrm{kHz}$ and the axial frequency is $0.22 \mathrm{kHz}$.

For ${ }^{6} \mathrm{Li}$ in a $\mathrm{CO}_{2}$ laser trap, both the excited and the ground states are attracted to the well. The excited state static polarizability is $\alpha_{p}=18.9 \times 10^{-24} \mathrm{~cm}^{3}$ [17], only $20 \%$ less than that of the ground state. With a ground state well depth of $8 \mathrm{MHz}$, the frequency of the first resonance transition in the trap is shifted by only $1.6 \mathrm{MHz}$ at the center of the trap and thus does not significantly alter the operation of the magneto-optical trap (MOT) from which the trap is loaded.

The optical scattering rate $R_{s}$ in the $\mathrm{CO}_{2}$ laser trap arises from Larmor scattering [4] and can be written as $R_{s}=\sigma_{S} I /(\hbar c k)$, where the Larmor scattering cross section $\sigma_{S}$ is

$$
\sigma_{S}=\frac{8 \pi}{3} \alpha_{g}^{2} k^{4}
$$

Here, $k=2 \pi / \lambda_{\mathrm{CO}_{2}}$. Using $\alpha_{g}=24.3 \times 10^{-24} \mathrm{~cm}^{3}$ yields $\sigma_{S}=5.9 \times 10^{-30} \mathrm{~cm}^{2}$. At $1.0 \mathrm{MW} / \mathrm{cm}^{2}$, the scattering rate for lithium is then $2.9 \times 10^{-4} / \mathrm{sec}$, corresponding to a scattering time of $\simeq 3400 \mathrm{sec}$ for one photon per atom. As a result, the recoil heating rate is negligible.

Heating can arise from laser intensity noise and beam pointing fluctuations 11, 12]. For simplicity, we estimate the noise-induced heating rates for our trap using a harmonic oscillator approximation which is valid for atoms 
near the bottom of the well. This provides only a rough estimate of the expected heating rates in the gaussian well, since the trap oscillation frequency decreases as the energy approaches the top of the well. A detailed discussion of noise-induced heating in gaussian potential wells will be given in a future publication. In the harmonic oscillator approximation, intensity noise causes parametric heating and an exponential increase in the average energy for each direction of oscillation, $\langle\dot{E}\rangle=\Gamma\langle E\rangle$, where the rate constant in $\mathrm{sec}^{-1}$ is

$$
\Gamma=\pi^{2} \nu^{2} S_{I}(2 \nu)
$$

Here $\nu$ is a trap oscillation frequency and $S_{I}(2 \nu)$ is the power spectrum of the fractional intensity noise in fraction ${ }^{2} / \mathrm{Hz}$. For our $\mathrm{CO}_{2}$ laser, $S_{I}(9.4 \mathrm{kHz}) \leq 1.0 \times$ $10^{-13} / \mathrm{Hz}$, where it is comparable to the detector noise. This is three orders of magnitude lower than that measured for an argon ion laser [11]. The corresponding heating time for radial oscillation in our trap at $\nu=4.7 \mathrm{kHz}$ is $\Gamma^{-1} \geq 4.6 \times 10^{4}$ sec. For the axial oscillation, $\nu=220$ $\mathrm{Hz}, S_{I}(440 \mathrm{~Hz}) \simeq 1.1 \times 10^{-11} / \mathrm{Hz}$ and $\Gamma^{-1} \simeq 2 \times 10^{5} \mathrm{sec}$.

Fluctuations in the position of the trapping laser beam cause a constant heating rate $\langle\dot{E}\rangle=\dot{Q}$, where

$$
\dot{Q}=4 \pi^{4} M \nu^{4} S_{x}(\nu) \text {. }
$$

Here $M$ is the atom mass and $S_{x}$ is the position noise power spectrum in $\mathrm{cm}^{2} / \mathrm{Hz}$ at the trap focus. For ${ }^{6} \mathrm{Li}$, one obtains $\dot{Q}(\mathrm{nK} / \mathrm{s})=2.8 \times 10^{-4} \nu^{4}(H z) S_{x}\left(\mu \mathrm{m}^{2} / \mathrm{Hz}\right)$. Position noise only couples directly to the radial motion where $\nu \simeq 4.7 \mathrm{kHz}$. For our laser, $S_{x}(4.7 \mathrm{kHz}) \leq 3.4 \times$ $10^{-10} \mu \mathrm{m}^{2} / \mathrm{Hz}$, where the upper bound is determined by the noise floor for our detection method. This yields $\dot{Q} \leq$ $46 \mathrm{nK} / \mathrm{s}$. Hence, we expect the trap lifetime to be limited by the background pressure of our UHV system.

The expected number of trapped atoms $N_{T}$ can be estimated as follows. We take the trapping potential to be approximately gaussian in three dimensions:

$$
U(\vec{x})=-U_{0} \exp \left(-x^{2} / a^{2}-y^{2} / b^{2}-z^{2} / z_{o}^{2}\right),
$$

where $a=b=a_{f} / \sqrt{2}$ is the intensity $1 /$ e radius and $z_{o}$ is the Rayleigh length. Here, the lorentzian dependence of the trap beam intensity on the axial position $z$ is approximated by a gaussian dependence on $z$.

We assume that after a sufficient loading time, atoms in the $\mathrm{CO}_{2}$ laser trap will come into thermal and diffusive equilibrium with the MOT atoms that serve as a reservoir 18]. The density of states in the gaussian trap and the occupation number then determine the number of trapped atoms, which takes the form

$$
N_{T}=n V_{F O R T} F\left[U_{0} /\left(k_{B} T\right)\right] .
$$

Here the volume of the $\mathrm{CO}_{2}$ laser trap is defined as $V_{F O R T}=a^{2} z_{o} \pi^{3 / 2}$. Hence, $n V_{F O R T}$ is the total number of atoms contained in the volume of the FORT at the MOT density $n$.
$F(q)$ determines the number of trapped atoms compared to the total number contained in the FORT volume at the MOT density. It is a function only of the ratio of the well depth to the MOT temperature, $q \equiv U_{0} /\left(k_{B} T\right)$ :

$$
F(q)=\frac{q^{3 / 2}}{2} \int_{0}^{1} d x x^{2} g(x) \exp [q(1-x)]
$$

Here $g(x)$ is the ratio of the density of states for a gaussian well to that of a three dimensional harmonic well:

$g(x)=\frac{\beta^{3 / 2}(1-x)^{1 / 2}}{x^{2}} \frac{16}{\pi} \int_{0}^{1} d u u^{2} \sqrt{\exp \left[\beta\left(1-u^{2}\right)\right]-1}$

where $\beta \equiv-\ln (1-x)$. The variable $x=\left(E+U_{0}\right) / U_{0}$ is the energy of the atom relative to the bottom of the well in units of the well depth, where $-U_{0} \leq E \leq 0$, and $g(0)=1$. For our MOT, the typical temperature is $1 \mathrm{mK}$, $n \simeq 10^{11} / \mathrm{cm}^{3}$, and $n V_{F O R T}=5 \times 10^{5}$ atoms. Using the well depth of $U_{0}=0.4 \mathrm{mK}$ in Eq. 6 shows that $N_{T}$ is of the order of $6 \times 10^{4}$ atoms. Much higher numbers are obtainable for a deeper well at lower temperature.

The experiments employ a custom-built, stable $\mathrm{CO}_{2}$ laser. High-voltage power supplies, rated at $10^{-6}$ fractional stability at full voltage, proper electrode design, and negligible plasma noise enable highly stable current. Heavy mechanical construction, along with thermally and acoustically shielded invar rods, reduces vibration. The laser produces $56 \mathrm{~W}$ in an excellent $\mathrm{TEM}_{00}$ mode.

The $\mathrm{CO}_{2}$ laser beam is expanded using a $\mathrm{ZnSe}$ telescope. It is focused through a double-sealed, differentially-pumped, $5 \mathrm{~cm}$ diameter ZnSe window into a UHV system. The vacuum is maintained at $\simeq 10^{-11}$ Torr by a titanium sublimation pump. The trap is at the focus of a $19 \mathrm{~cm}$ focal length ZnSe lens.

The trap is continuously loaded from a ${ }^{6} \mathrm{Li}$ MOT employing a standard $\sigma_{ \pm}$configuration [19] with three orthogonal pairs of counterpropagating, oppositelypolarized $671 \mathrm{~nm}$ laser beams, each $2.5 \mathrm{~cm}$ in diameter and $8 \mathrm{~mW}$. Power is supplied by a Coherent 699 dye laser that generates $700 \mathrm{~mW}$. The MOT magnetic field gradient is $15 \mathrm{G} / \mathrm{cm}(7.5 \mathrm{G} / \mathrm{cm})$ along the radial (axial) directions of the trap. The MOT is loaded from a multicoil Zeeman slower system 20] that employs a differentially pumped recirculating oven source [21]. Using a calibrated photomultiplier, the MOT is estimated to trap approximately $10^{8}{ }^{6} \mathrm{Li}$ atoms. The MOT volume is found to be $\simeq 1 \mathrm{~mm}^{3}$. This yields a density of $10^{11} / \mathrm{cm}^{3}$, consistent with that obtained for lithium in other experiments 22,23. Using time-of-flight methods, we find typical MOT temperatures of $1 \mathrm{mK}$.

We initially align the $\mathrm{CO}_{2}$ laser trap with the MOT by using split-image detection of the fluorescence at $671 \mathrm{~nm}$ to position the focusing $\mathrm{ZnSe}$ lens in the axial direction. The focal point for the trapping beam is positioned in the center of the MOT, taking into account the difference in the index of refraction of the optics at $671 \mathrm{~nm}$ 
and $10.6 \mu \mathrm{m}$. A $671 \mathrm{~nm}$ laser beam is aligned on top of the $\mathrm{CO}_{2}$ laser beam to align the transverse position of the focal point in the MOT. Since the Rayleigh length is short and the focus is tight, this method does not reliably locate the actual focus of the $\mathrm{CO}_{2}$ beam. Hence, a spectroscopic diagnostic based on the light shift induced by the $\mathrm{CO}_{2}$ laser is employed for final alignment of the trapping beam.

While the near equality of the Li excited and ground state polarizabilities is ideal for continuous loading from the MOT, it makes locating the $\mathrm{CO}_{2}$ laser focus in the MOT by light shift methods quite difficult. To circumvent this problem, a dye laser at $610 \mathrm{~nm}$ is used to excite the 2p-3d transition for diagnostics. At the $10.6 \mu \mathrm{m}$ $\mathrm{CO}_{2}$ laser wavelength, we estimate that the $3 \mathrm{~d}$ state has a scalar polarizablity of approximately $700 \times 10^{-24} \mathrm{~cm}^{3}$ [24], nearly 30 times that of the $2 \mathrm{~s}$ or $2 \mathrm{p}$ state. In the focus of the $\mathrm{CO}_{2}$ laser, the corresponding light shift is $\simeq-300$ $\mathrm{MHz}$. Chopping the $\mathrm{CO}_{2}$ laser beam at $2 \mathrm{kHz}$ and using lock-in detection of fluorescence at $610 \mathrm{~nm}$ yields a twopeaked light shift spectrum. This two-peaked structure arises because the lock-in yields the difference between signals with the $\mathrm{CO}_{2}$ laser blocked and unblocked. At the ideal focusing lens position, the amplitude and the frequency separation of these peaks are maximized. Optical alignment remains unchanged for months after this procedure.

Measurement of the trapped atom number versus time is accomplished by monitoring the fluorescence at 671 nm induced by a pulsed, retroreflected probe/repumper beam (1 $\mathrm{mW}, 2 \mathrm{~mm}$ diameter). The probe is doubleblinded by acousto-optic $(\mathrm{A} / \mathrm{O})$ modulators to minimize trap loss arising from probe light leakage. The loading sequence is as follows: First, the $\mathrm{CO}_{2}$ laser trap is continuously loaded from the MOT for 10 seconds. This provides adequate time for the MOT to load from the Zeeman slower. Then the MOT repumping beam is turned off, so that atoms in the upper $F=3 / 2$ hyperfine state are optically pumped into the lower $F=1 / 2, M= \pm 1 / 2$ states. After $25 \mu \mathrm{sec}$, the optical MOT beams are turned off using $\mathrm{A} / \mathrm{O}$ modulators, and a mechanical shutter in front of the dye laser is closed within $1 \mathrm{~ms}$ to eliminate all MOT light at $671 \mathrm{~nm}$. The MOT gradient magnets are turned off within $0.2 \mathrm{~ms}$. After a predetermined time interval between 0 and $600 \mathrm{sec}$, the probe beam is pulsed to yield a fluorescence signal proportional to the number of trapped atoms. The detection system is calibrated and the solid angle is estimated to determine the atom number. Typical trapped atom numbers measured in our initial experiments are $\simeq 2.3 \times 10^{4}$. This corresponds to the predictions of Eq. 6 for a well depth of $0.25 \mathrm{mK}$. Since we expect the potential of the MOT gradient magnet to lower the effective well depth from $0.4 \mathrm{mK}$ by $\simeq 0.15 \mathrm{mK}$ during loading, the measured trap number is consistent with our predictions.

Fig. 1 shows the decay of the trapped atom number on a time scale of $0-600$ seconds. Each data point is the mean obtained from four separate measurement se- quences through the complete decay curve. The error bars are the standard deviation from the mean. Atoms in the $F=1 / 2$ state exhibit a single exponential decay with a time constant of $297 \mathrm{sec}$, clearly demonstrating the potential of this system for measurements on a long time scale.

We have observed that an initial $10-15 \%$ decrease in the signal can occur during the first second. This may arise from inelastic collisions between atoms in the $F=$ $1 / 2$ state with atoms that are not optically pumped out of the upper $F=3 / 2$ state. During optical pumping, fluorescence from the $\mathrm{F}=3 / 2$ state decays in $\simeq 5 \mu \mathrm{sec}$ to $\mathrm{a} \simeq 5 \%$ level which persists for a few milliseconds, consistent with a residual $F=3 / 2$ population.

The lifetime of atoms in the $F=1 / 2$ state can be limited by processes that cause heating or direct loss. If we attribute the trap lifetime entirely to residual heating, the heating rate from all sources would be at most $400 \mu \mathrm{K} / 300 \mathrm{sec} \simeq 1 \mu \mathrm{K} / \mathrm{sec}$, which is quite small. However, if the loss were due to heating, one would expect a multimodal decay curve, analogous to that predicted in Ref. 12. Instead, we observe a single exponential decay as expected for direct loss mechanisms, such as collisions with background gas atoms or optical pumping by background light at $671 \mathrm{~nm}$ (into the unstable $F=3 / 2$ state). If we assume that the lifetime is background gas limited and that $\mathrm{Li}$ is the dominant constituent, the measured lifetime of $297 \mathrm{sec}$ is consistent with a pressure of $\simeq 10^{-11}$ Torr.

The long lifetime of the $F=1 / 2$ state is expected, based on the prediction of a negligible s-wave elastic scattering length $(<<1$ Bohr) at zero magnetic field [25]. Hence, spontaneous evaporation should not occur. We have made a preliminary measurement of trap loss arising from inelastic collisions when the $F=3 / 2$ state is occupied. This is accomplished by omitting the optical pumping step in the loading sequence described above. The trap is found to decay with a $1 /$ e time $<1$ sec when $2.3 \times 10^{4}$ atoms are loaded (density $\simeq 10^{9} / \mathrm{cm}^{3}$ ). A detailed study of elastic and inelastic collisions at low magnetic field is in progress.

In conclusion, we have demonstrated a $300 \mathrm{sec} 1 / \mathrm{e}$ lifetime for lithium fermions in an ultrastable $\mathrm{CO}_{2}$ laser trap with a well depth of $0.4 \mathrm{mK}$. By using an improved aspherical lens system, an increase in trap depth to $1 \mathrm{mK}$ is achievable. Further, Eq. 6 shows that, if the MOT temperature is reduced to $0.25 \mathrm{mK}$, more than $10^{6}$ atoms can be trapped in a $1 \mathrm{mK}$ deep well. Since the ground and excited state trapping potentials are nearly identical, exploration of optical cooling schemes may be particularly fruitful in this system. Currently, we are exploring ${ }^{6} \mathrm{Li}$ as a fundamental example of a cold, weaklyinteracting fermi gas. By trapping multiple hyperfine states, it will be possible to study both elastic and inelastic collisions between fermions. The combination of long storage times and tight confinement obtainable with the $\mathrm{CO}_{2}$ laser trap, as well as the anomalously large scattering lengths for ${ }^{6} \mathrm{Li}$ [26,27], make this system an excellent 
candidate for evaporative cooling and potential observation of a Bardeen-Cooper-Schrieffer transition. Further, this system is well suited for exploring novel wave optics of atoms and molecules, such as coherent changes of statistics by transitions between free fermionic atoms and bosonic molecules, analogous to free to bound transitions for bosonic atoms 28 .

We thank Dr. R. Hulet for stimulating conversations regarding this work. We are indebted to Dr. C. Primmerman and Dr. R. Heinrichs of MIT Lincoln Laboratory for the loan of two stable high voltage power supplies and to Dr. K. Evenson of NIST, Boulder for suggestions regarding the laser design. This research has been supported by the Army Research Office and the National Science Foundation.

${ }^{\dagger}$ Permanent Address, Department of Electrical Engineering and Computer Science, MIT, Cambridge, MA 02139 .

[1] See, D. J. Wineland and W. M. Itano, Phys. Rev. A 20, 1521 (1979) and references therein; J. P. Gordon and A. Ashkin, Phys. Rev. A21, 1606 (1980).

[2] J. D. Miller, R. A. Cline, and D. J. Heinzen, Phys. Rev. A 47, R4567 (1993).

[3] S. Rolston et al., in Proceedings of the 1992 Shanghai International Symposium on Quantum Optics, ed. Yuzhu Wang, Yiqiu Wang and Z. Wang [Proc. SPIE 1726, 205 (1992)].

[4] H. J. Lee et al., At. Phys. 14, 258 (1995).

[5] C. S. Adams et al., Phys. Rev. Lett. 74, 3577 (1995).

[6] D. M. Stamper-Kurn et al., Phys. Rev. Lett. 80, 2027 (1998).

[7] T. Takekoshi and R. J. Knize, Opt. Lett. 21, 77 (1996).

[8] M. V. Romalis, and E. N. Fortson, to appear in Phys. Rev. A 59 (1999); M. Bijlsma, B. J. Verhaar, and D. J. Heinzen, Phys. Rev. A 49, R4285 (1994); N. Davidson et al., Phys. Rev. Lett. 74, 1311 (1995).

[9] H. J. Lee, C. S. Adams, M. Kasevich, and S. Chu, Phys. Rev. Lett. 76, 2658 (1996).

[10] C. S. Adams and E. Riis, Prog. Quant. Elec. 21, 1-79 (1997).

[11] T. A. Savard, K. M. O'Hara, and J. E. Thomas, Phys. Rev. A 56, R1095 (1997).

[12] M. E. Gehm et al., Phys. Rev. A 58, 3914 (1998).

[13] C. Freed, Ultrastable $\mathrm{CO}_{2}$ lasers (Lincoln Laboratory, Bedford, MA, 1990) Vol. 3, 479-500.

[14] J. E. Thomas et al., Rev. Sci. Instrum. 51, 240 (1980).

[15] C. R. Monroe et al., Phys. Rev. Lett. 70, 414 (1993).

[16] The significance of quantum diffractive background gas collisions for heating and loss in shallow traps is discussed by S. Bali, K. M. O'Hara, M. E. Gehm, S. R. Granade, and J. E. Thomas, Phys. Rev. A, to be published.

[17] L. Windholz et al., Phys. Rev. A 46, 5812 (1992).

[18] A model of the spatial loading dynamics of a MOT- loaded gaussian laser beam trap will be discussed elsewhere.

[19] E. Raab et al., Phys. Rev. Lett. 59, 2631 (1987).

[20] T. A. Savard et al., Quantum Electronics and Laser Science Conference, Vol. 12, 1997 OSA Technical Digest Series (Optical Society of America, Washington, D.C., 1997), pp. 107-108.

[21] The design of the recirculating oven source was suggested to us by R. Hulet, Rice University.

[22] J. Kawanaka et al., Phys. Rev. A 48, R883 (1993); Z. Lin et al., Jap. Jour. Appl. Phys. 30, L1324 (1991).

[23] B. P. Anderson, T. L. Gustavson, and M. A. Kasevich, Phys. Rev. A 53, R3727 (1996).

[24] Atomic Transition Probabilities, Vol.I, eds. W. L. Wiese, M. W. Smith, and B. M. Glennon, National Standard Reference Data Series-NBS 4 (Washington, DC, 1966), pp.16-18.

[25] M. Houbiers, H. T. C. Stoof, W. I. McAlexander, and R. G. Hulet, Phys. Rev. A 57, R1497 (1998).

[26] H. T. C. Stoof, M. Houbiers, C. A. Sackett, and R. G. Hulet, Phys. Rev. Lett. 76, 10 (1996).

[27] M. Houbiers et al., Phys. Rev. A 56, 4864 (1998).

[28] D. J. Heinzen, P. D. Drummond, and K. V. Kherunstyan, Bull. Am. Phys. Soc.,44, 1007 (1999); J. Javanainen and M. Mackie, Bull. Am. Phys. Soc., 44, 1007 (1999).

FIG. 1. Trapped number of atoms versus time for an ultrastable $\mathrm{CO}_{2}$ laser trap. The solid line is a single exponential fit, $N(t)=A \exp (-t / \tau)$, and gives $\tau=297 \mathrm{sec}$. We believe that a small fraction of atoms are lost at short times $\leq 1 \mathrm{sec}$, (see insert, 0-10 sec) from collisions with atoms that remain in the $F=3 / 2$ state after optical pumping. Hence, the first two points at 0.1 and $0.3 \mathrm{sec}$ are neglected in the fit. The trap lifetime for the remaining $F=1 / 2$ atoms is 297 seconds, to our knowledge the longest ever obtained with an all-optical trap. 